\title{
Comparing Optical Turbulence Using a Pair of Sonic Anemometers
}

\author{
N. P Mashaba and D. J Griffith
}

\begin{abstract}
Turbulence is regarded as one of the most deleterious effects on imaging systems. The CSIR have an optical test facility, used to test image quality of surveillance systems, however, the temporal distribution of the near field turbulence is unknown. Here, we deploy a pair of ultrasonic anemometers in the vicinity of the optical field test facility and we measure the wind speed of the airflow. We explain how to extract all the parameters from the real-world data and we calculate turbulence. Lastly, we will present results and indicate the limitations that atmospheric turbulence may contribute towards degrading the quality of images from imaging systems.
\end{abstract}

Keywords - Atmospheric turbulence, optical surveillance systems, sonic anemometer.

\section{INTRODUCTION}

Optical surveillance systems have gained popularity; mainly in military, private enterprise and commercial. Atmospheric turbulence is a major limitation on the range at which optical surveillance systems can be effective. Detection, recognition and identification (DRI) range of medium to long range optical surveillance systems is strongly impacted by atmospheric turbulence [1], [2]. Scintillometry and point source imaging have been the main techniques for turbulence strength measurements using optical principles [3]. However, these techniques are limited to providing a measure of turbulence strength that is integrated over the entire optical sightline. Importantly, the turbulence near the input aperture of an imaging system ("near-field" turbulence) is particularly damaging to image quality whereas path-integrated measurements of turbulence do not reveal the location of the most turbulent air. In contrast to the optical methods, the technique of sonic anemometry allows for localised measurements of turbulence strength, permitting measurements in the near-field.

Our research group operates an optical test facility (situated in the roof of building 44 at the CSIR) from which many optical surveillance systems are being evaluated. The temporal distribution of turbulence is unknown and therefore, the effects of optical turbulence in the area surrounding the facility are also unknown. Studies have shown that turbulence strength is highly variable over diurnal and seasonal cycles and from point

Manuscript received 10 June, 2020

This work was supported by the Optronics Sensor Systems (OSS) group at the CSIR.

N. P Mashaba is with The Optronics Sensor Systems department at The Council for Scientific and Industrial Research (CSIR), Defence and Security Cluster, P. O. Box 395, Pretoria, South Africa .

D. J Griffith is with Hensoldt Optronics, Nellmapius Dr, Highveld, Centurion, 0157 . to point in any environment [4]. Understanding of the temporal distribution of turbulence strength in an environment allows for possible avoidance and mitigation approaches and strategies. Here we determine the temporal distribution of turbulence by installing a pair of sonic anemometers at two different point, shown above. A need for looking at the temporal distribution of turbulence in the near-field have to be pursue in order to improve the quality of our imaging systems and also be aware of strong and weak weather conditions.

Near-field turbulence analysis may allow for engineering manipulation, whereas far-field turbulence is generally outside engineering control. For this reason, it becomes interesting and important to have methods of small volume turbulence measurements. A possible pair of Gill HS-50 sonic anemometers is proposed to be deployed at various points in the roof laboratory to obtain long-term turbulence strength measurements at high temporal resolution. In this paper, we install the pair of sonic anemometers and we measure the velocity of the airflow as it changes with time. We will extract turbulence parameters and compare the results from the two instruments. The final phase will involve a way forward and conclusions based on the work done.

\section{THE EQUIPMENT}

\section{A. Sonic anemometers}

A sonic anemometer is a solid-state ultrasonic instrument having the ability to measure wind velocities in three orthogonal axes (U, V, and $\mathrm{W})$ and supplies sonic temperature. The instrument consists of three pairs of transducers orientated at multiple angles across the measurement volume. Each pair of transducers act alternately in the transmitting and receiving modes, sending ultrasonic pulses between the pair. The anemometers are logged at the maximum rate of 50

$\mathrm{Hz}$ (internal block averages over the raw internal sampling rate of $100 \mathrm{~Hz}$ ). This rate is sufficient to resolve the small scale eddies in the atmosphere as well as provide the 3D wind velocity. The sensor head of the Gill 3D sonic anemometer comprises of an arrangement of three pairs of transducers orientated at multiple angles across the measurement volume. The sonic anemometer principle and instrument present a unique method of measuring the strength of atmospheric turbulence in a small volume. This can be considered a point measurement of turbulence strength in comparison to scintillometry and point source imaging, which measure the integrated effect of turbulence along the full optical sightline. Probing of local turbulence strength using sonic anemometry is 
therefore a complementary method to the optical techniques and allows for more detailed establishment of the turbulence picture in a specific environment and circumstance. This is especially important when attempting to discover the near-field turbulence strength, which is particularly degrading the imaging quality of an optical system. Fig. 1(a) shows the layout of the instrument head. The vertical stiffener member is used as a reference in order to align the instrument head.

Fig. 1(b) shows an example of a pair of transducers, sending and receiving pulses to one another. The transducers are separated by distance 1 and the travelling time for each pulse is measured ( $\mathrm{t} 1$ and $\mathrm{t} 2$ ). With measuring the times of flight of the ultrasonic pulses in both directions along the path, the wind speed, $v$ of the airflow can be can be written as

$$
v=\frac{l}{2}\left(\frac{1}{t_{1}}-\frac{1}{t_{2}}\right) \text {. }
$$

The sonic temperature, Ts can be calculated using [3], [5]

$$
T_{s}=\frac{l^{2}}{1612}\left(\frac{1}{t_{1}}+\frac{1}{t_{2}}\right)+\frac{1}{403} v_{U}^{2}+v_{V}^{2}
$$

\section{B. The platinum resistance thermometer (PRT) sensor}

An additional thermometer is commonly installed next to the sonic anemometer to gather the absolute temperature. Here, we review the Pt100 outdoor / cold store temperature sensors type of PRT. The Pt100 has a resistance value of $100.0 \Omega$ at $0 \circ \mathrm{C}$ and a change of $38.50 \Omega$ between 0 and $100 \circ \mathrm{C}[6]$.
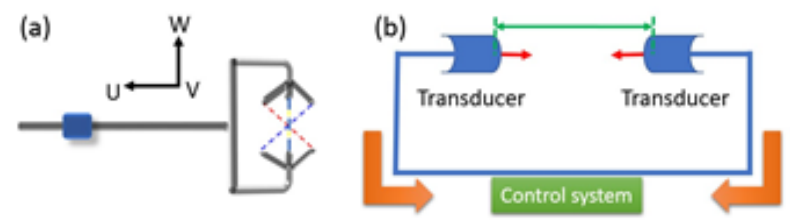

Fig. 1. (a) Pictures of the Gill HS-50 sonic anemometer. The three pairs of transducers are shown, where each transducer alternately acts as a transmitter and receiver. (b) The time of flight technical diagram. The transmitted and received pulse times are taken for a sound pulse which pass along one axis, is measured in both directions.

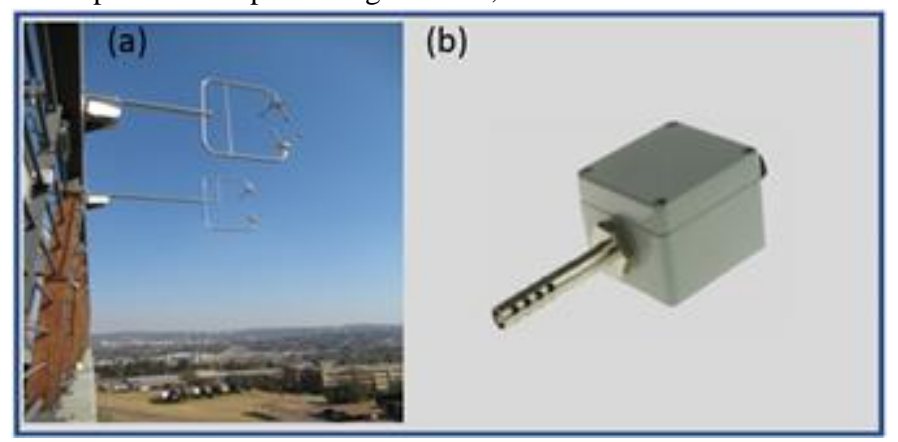

Fig. 2. (a) A pair of sonic anemometers installed on the rail of the optical test facility at the CSIR. (b) A photo of a PRT sensor.

\section{OUTDOOR TESTING}

The type of sonic anemometer that we installed at the CSIR's optical test facility is the Gill HS-50, shown in Fig. 2(a). The pair of ultrasonic anemometers was positioned 1 meter away from the other in the optical field open facility. The anemometer is attached to a sonic temperature sensor, a PRT sensor (shown in 2(b)) and they are connected to an electronic box system and read out data. The output is the measurement time, the three dimensional (3d) wind velocity, sonic temperature and absolute temperature. The measurement time has a resolution of $100 \mathrm{~Hz}$. We calculated the velocities of three vector components (U, V, and $\mathrm{W}$ ), using the collected 2019 four months (Jan, Feb, May and June) data by the sonic anemometer. We selected a specific date from these four months.

Fig. 3(a) and (b) shows the calculated wind speeds from the data of the pair of sonic anemometers. The results were recorded over a 24 hours cycle, from morning until midnight. The results show that the airflow is almost constant in the morning and at night. As the wind velocity starts picking up in the afternoon, the friction velocity becomes high and the velocity of the components varies in the opposite directions (the $\mathrm{U}$ and $\mathrm{W}$ vector). We were able to compute the mean wind, shown in Fig. 3(c) and (d). All these parameters are important for computing the refractive index structure function, $C_{a}^{2}$. During this period, the average pressure is also measured.

The sonic temperature is measured in kelvin directly by the sonic anemometer data acquisition software and is based on the average speed of sound, c, measured in both directions on the three axes. A fast response temperature related measurement is also generated in the process using a PRT sensor, which forms the basis of the turbulence measurements. Data obtained from the instruments allows a range of turbulence parameters and fluxes to be derived, using eddy correlation methods, in addition to obtaining various wind statistics which ease research. Fig. 4 (a) and (b) shows the sonic temperatures and the absolute temperatures using a spefic day of January and February data, measured over a 24 hour cycle. The temperature difference from the pair of sonic anemometer, from point A to point $B$ is not much of a big difference. Interestingly, it can be seen in Fig. 4(c) and (d) that the sonic and absolute temperature
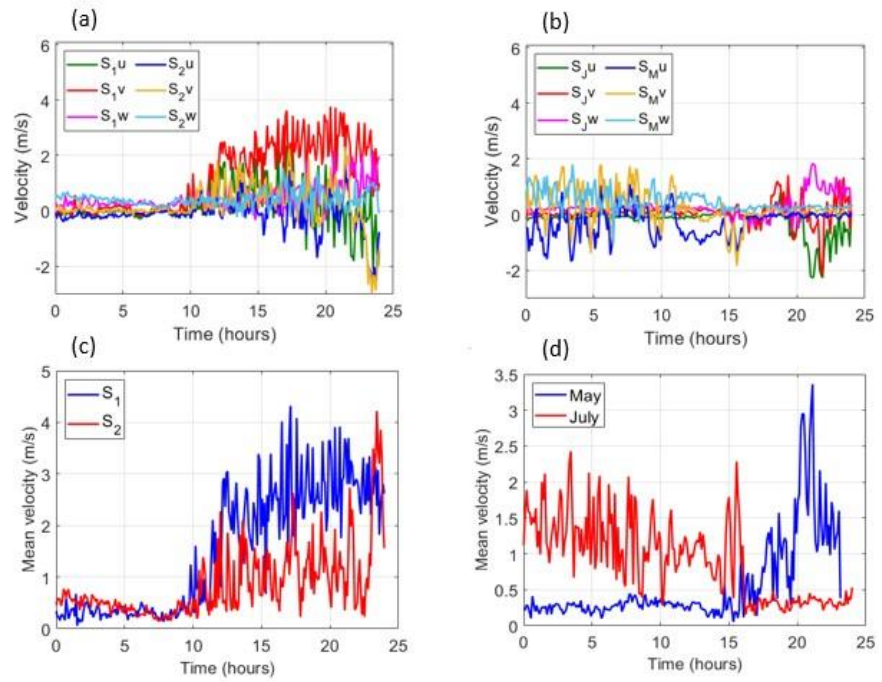

Fig. 3. (a)-(b) Velocity results of the pair of sonic anemometers. (c)-(d) Mean velocities of the two instruments. (c). 
changes a lot more from season to season. The samples that we made here, were May and July. Here we used a single sonic anemometer. (a)

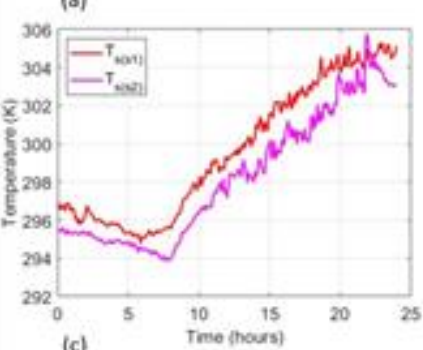

(c)

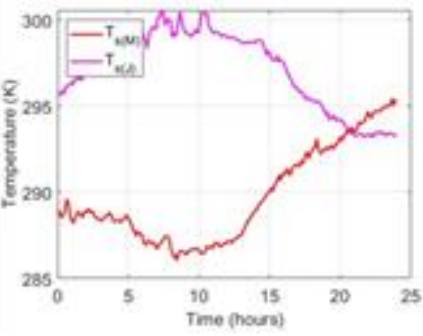

(b)

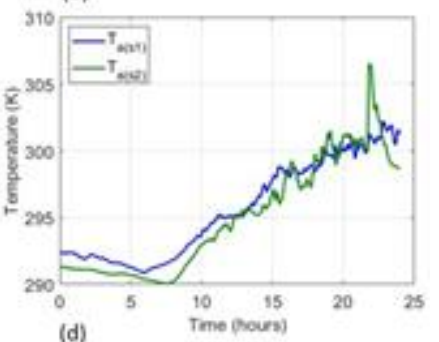

(d)

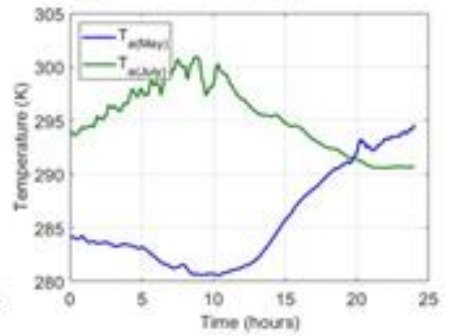

Fig. 4. Sonic temperatures, taken by a pair of sonic anemometers

From sonic temperature measurements, we can estimate turbulence. In optical terms, the most common measure of turbulence strength is the refractive index structure function constant, $C_{a}^{2}$. The intermediate, to which this is strongly related [7] is the structure function constant for temperature $C_{T}^{2}$, which is related to the sonic temperature statistics

$$
C_{T}^{2}=13.67 \times S_{T}(f) f^{5 / 3} U^{2 / 3},
$$

where $\mathrm{S}_{T}(\boldsymbol{f})$ is the temperature power spectrum over temporal frequency $\boldsymbol{f}$ and $\boldsymbol{U}$ is the mean wind speed over the period of the spectral analysis (anything from 1 minute to as much as 20 minutes).
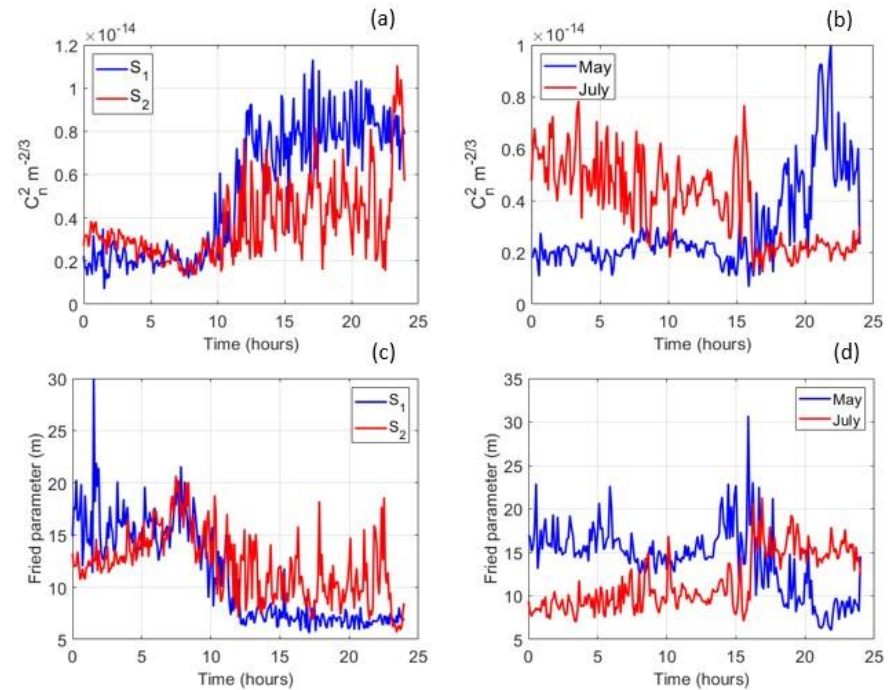

Fig. 5. (a) Refractive index structure function of the pair of sonic anemometers. (b) Refractive index structure function results from a single sonic anemometer, recorded on different months and a day.

(c)-(d) fried parameter results.

Systematic error creeps into the experimental results because of uncontrolled and often cryptic deterministic processes. Errors in most cases emanate from uncontrollable conditions or inaccuracy of instruments due to malfunction and lack of calibration. In practice, a number of corrections to the temperature power spectrum are required before application to the relationship in Eq. (3). In this section, we describe and estimate the different types of corrections that are required to transform the sonic-anemometer's data into real single point measurements of temperature and wind velocity.

\section{DERIVING ThE REFRACTIVE INDEX STRUCTURE FunCTION}

The refractive-index structure constant $C^{2}$ is a measure of the strength of the fluctuations in the refractive index. The behaviour of point along propagation path can be deduced from the temperature structure function obtained from point measurements of the mean- square temperature difference of two fine wire thermometers [6]. The refractive index structure constant is obtained from this equation

$$
C_{n}^{2}=\left(79 \times 10^{-6} \frac{P}{T^{2}}\right)^{2} C_{T}^{2}
$$

Where $\mathrm{P}$ is the pressure, and $\mathrm{T}$ is the absolute temperature. Fig. 5(a)- (b) shows the refractive index structure function for the pair of the instrument. The values are in the order of $10^{-14}$ $\mathrm{m}^{-2 / 3}$ which means the turbulence strength in the optical lab facility is medium. For a strong turbulence, the values of the refractive index structure function have to be in the order of $10^{-13} \mathrm{~m}^{-2 / 3}$. While Fig. 5(c)-(d) shows the coherence length (Fried parameter, $r_{0}$ ), being the measure of the quality of optical transmission through the atmosphere. The results show medium amount of turbulence scales.

\section{CONCLUSION}

In this work, we have measured small volumes of the velocity airflow. The results have shown that a pair of sonic anemometers, separated by smaller distances does not produce the same results. We have demonstrated the proof of principle of turbulence. The strength of turbulence was medium and it can be controlled. For future work will be proposed to correct for near-field turbulence, using adaptive optics.

\section{REFERENCES}

[1] J. Dijk, K. Schutte, and R. P. Nieuwenhuizen, "Turbulence mitigation methods for sea scenarios," in Electro-Optical and Infrared Systems: Technology and Applications XIII, vol. 9987, p. 99870E, International Society for Optics and Photonics, 2016.

https://doi.org/10.1117/12.2243165

[2] R. P. Nieuwenhuizen, A. W. van Eekeren, J. Dijk, and K. Schutte, "Dy- namic turbulence mitigation with large moving objects," in Electro-Optical and Infrared Systems: Technology and Applications XIV, vol. 10433, p. 104330S, International Society for Optics and Photonics, 2017. https://doi.org/10.1117/12.2277840

[3] J. C. Kaimal and J. J. Finnigan, Atmospheric boundary layer flows: their structure and measurement. Oxford university press, 1994.

[4] J. R. Garratt, "The atmospheric boundary layer," Earth-Science Reviews, vol. 37, no. 1-2, pp. 89-134, 1994. https://doi.org/10.1016/0012-8252(94)90026-4

[5] J. Kaimal and J. Gaynor, "Another look at sonic thermometry," Boundary-Layer Meteorology, vol. 56, no. 4, pp. 401-410, 1991. https://doi.org/10.1007/BF00119215

[6] M. A. L. Regalado, "Evaluation of the contribution to uncertainty due to joule self-heating effect in the temperature measurement using platinum resistance thermometers.,"

[7] L. C. Andrews and R. L. Phillips, "Laser beam propagation through random media," SPIE, 2005.

https://doi.org/10.1117/3.626196 